\title{
An audit of nutritional care in patients receiving parenteral nutrition; a response to the National Clinical Enquiry into Patient Outcome and Death "A Mixed Bag” 2010
}

\author{
J. Saunders, R. Thornber, T. Smith, Z. Leach, L. Buse, L. Bakewell, P. Austin and M. Stroud \\ Southampton NIHR Nutrition, Diet and Lifestyle BRU, Southampton General Hospital, Southampton SO16 6YD, UK
}

A National Confidential Enquiry into Patient Outcome and Death (NCEPOD) recently reported appalling national standards; $81 \%$ of patients failing to receive a good standard of care when cases were externally peer reviewed ${ }^{(1)}$. We audited the quality of care received by parentally fed patients at Southampton University Hospital Trust (SUHT), a specialist regional centre with an intestinal failure unit (IFU).

Data were collected prospectively from 50 patients over an 8 week period, based on a modified NCEPOD audit tool. An additional study to assess parenteral nutrition (PN) administration and accuracy of fluid balance recording in different ward areas was also undertaken. PN bags were weighed pre and post administration to calculate the volume patients received and compared with data recorded on fluid balance charts.

\begin{tabular}{lcc}
\hline Outcome & SUHT & NCEPOD \\
\hline Weight/MUST & $100 \%$ & $50 \%$ \\
Blood glucose monitoring & $90 \%$ & $48 \%$ \\
IFU & $95 \% *$ & \\
Non-IFU & $71 \%$ & $39 \%$ \\
Metabolic Complications & $28 \%$ & $26 \%$ \\
Catheter-related complications & $4 \%$ & 52.7 \\
NST involved in initiating PN & $90 \%$ & \\
* Significant IFU vs. Non-IFU $(\mathrm{p}<0.05)$. & &
\end{tabular}

\begin{tabular}{|c|c|c|c|c|c|c|}
\hline & $\begin{array}{l}\text { Number of } \\
\text { PN bags }\end{array}$ & $\begin{array}{l}\text { Mean volume } \\
\text { prescribed mls } \\
\text { (P) }\end{array}$ & $\begin{array}{c}\text { Mean volume } \\
\text { administered mls } \\
\text { (A) }\end{array}$ & $\begin{array}{l}\text { Mean volume } \\
\text { recorded mls } \\
(\mathrm{R})\end{array}$ & $\begin{array}{c}\text { Difference } \\
\text { A-P } \\
(\%)\end{array}$ & $\begin{array}{c}\text { Difference } \\
\text { A-R } \\
(\%)\end{array}$ \\
\hline IFU & 189 & 2118 & 2198 & 2100 & +3.5 & +5.1 \\
\hline Non- IFU & 41 & 1763 & 1866 & 1716 & +6.0 & +9.9 \\
\hline$P$ value & & & & & 0.23 & 0.03 \\
\hline
\end{tabular}

Higher standards of assessment and monitoring were seen than in national data. There were fewer metabolic complications and although a significant number of patients still had abnormal blood results, in reality these were probably related to the underlying disease process, rather than PN-related complications. None of the patients developed refeeding syndrome and there were far fewer catheter complications, probably because of the high nursing standards on the IFU. The nutrition support team (NST) were not involved in initiation of PN in $5(10 \%)$ of cases, all of which occurred out of hours, despite an existing Trust policy. When the NST were subsequently involved in these patients PN was felt to have been initiated inappropriately in 2 cases and consequently was stopped the next working day.

There was a trend towards better PN care on the IFU, with more precise PN administration, significantly more accurate fluid balance recording and monitoring for early metabolic complications. These changes were found despite the low patient numbers in the non-IFU group. However, it must be noted that the standards of monitoring in all clinical areas of the Trust were very high.

Our results demonstrate that high NCEPOD defined standards of PN care can be achieved and confirm the importance of an expert NST and the benefits of a specialist intestinal failure ward.

1. National Confidential Enquiry into Patient Outcome and Death (NCEPOD). A Mixed Bag. An enquiry into the care of hospital patients receiving parenteral nutrition 2010. 